\title{
Interactive system for geomagnetic data analysis
}

\author{
Igor Solovev ${ }^{*}$ \\ Institute of Cosmophysical Research and Radio Wave Propagation FEB RAS, 684034 Kamchatka \\ region, Elizovskiy district, Paratunka, Mirnaya str., 7, Russia
}

\begin{abstract}
The paper suggests the methods for analyzing geomagnetic field variations, which are implemented in "Aurora" software system for complex analysis of geophysical parameters. The software system allows one to perform a detailed magnetic data analysis. The methods allow one to estimate the intensity of geomagnetic perturbations and to allocate increased geomagnetic activity periods. The software system is publicly available

(http://aurorasa.ikir.ru:8580,

http://www.ikir.ru:8280/1saserver/MagneticPage.jsp).

This research was supported by the Russian Science Foundation (Project No. 14-11-00194).
\end{abstract}

\section{Introduction}

In this paper, we described software tools for geomagnetic data analysis, based on the application of modern mathematical methods and information technologies. Implemented software methods are aimed at studying of complex dynamic processes in the magnetosphere. The state of the magnetosphere is determined by the parameters of the interplanetary space, which has a negative effect on satellite operation, radio communications and other important aspects of human life. Therefore, this field of research is of great scientific and practical interest today $[1,2]$.

Variations of the Earth's magnetic field contain important information on the processes in the magnetosphere that occur during the period of high solar activity. Currently, methods [3-6] and application software for the processing and analysis of geophysical data are being developed. They provide the users with convenient tools for conducting experimental and theoretical studies (eg. http://www.cosmos.ru/magbase; http://matlab.izmiran.ru/magdata/, https://www.ngdc.noaa.gov/; http://smdc.sinp.msu.ru/). Over the past 20 years, the network of terrestrial magnetic observatories has increased significantly, and a large amount of information has been accumulated. But at the same time, there are still unsolved problems, such as digitization of previously accumulated magnetograms [7-8], development of automatic systems for data collection and for initial data processing [9-11]. Due to the continuous accumulation of data and the transition to a second registration format, the relevance of these tasks is growing.

Anthropogenic interference arising in a signal does not allow us to monitor and to assess the Earth's magnetic field automatically. The authors of the works [2, 9-11], on the

\footnotetext{
* Corresponding author: kamigsol@yandex.ru
} 
basis of DataMining applications, developed methods for automating the work of experts (creating so-called "electronic experts") to solve the problems of primary data processing, to detect industrial and physical anomalies and to form world databases. Moreover, an algorithm based on the application of continuous wavelet transform [12] and threshold functions, first proposed in [3], showed high efficiency in detection of industrial noise.

To use the geomagnetic data effectively and to obtain qualitatively new results, it is necessary to create adequate automated data processing and analysis systems. Difficulties in solving these problems are related to the nonstationary structure of the field variations, the presence of local features of various shapes and durations that carry important information about the state of the earth's magnetic field. Taking into account the complex structure of field variations, modern mathematical methods and technologies are currently used. Thus, for example, to solve the problems of automatic detection of the periods of magnetic storm initial phase [13], of removing noise, and excluding the periodic component of geomagnetic field variations caused by Earth rotation [14, 15], a wavelet transform was used. On the basis of this tool, the problem of automatic calculation of geomagnetic activity index [16] was also solved, which made it possible to reduce the error of its calculation in comparison with the traditional methods [17-19] by $20 \%$. When solving the problems of the modeling of field variations [20] and prediction of the travel time of the interplanetary shock wave [21], neural networks are applied. The developed approach allowed us to improve the quality of the magnetic storm forecasting technique (by $9 \%$ of cases) in comparison with the method based on the logistic regression model [21]. In the papers [3, 22 ], based on the combination of neural networks and wavelet transform, a method for automatic estimation of the state of the geomagnetic field was proposed, and it was implemented as a software application.

The algorithms presented in this paper are based on the proposed geomagnetic signal model [23, 24], including methods for identification of a typical field component and various scale disturbances during the periods of high solar activity. The obtained numerical solutions make it possible to distinguish periods of weak and strong geomagnetic activity and to estimate the intensity of geomagnetic disturbances. Based on the processing of 1second data, geomagnetic disturbances can be analyzed in the ranges of Pc1-Pc5 and Pi1Pi3 pulsations. We also constructed wavelet portrait of the magnetic storm which shows the distribution of the disturbance intensity along the analyzed meridian of observations.

Numerical solutions formed the basis of the developed software component of "Aurora" system, which is in open access at http://aurorasa.ikir.ru:8580 (mirror linkhttp://aurorasa2.ikir.ru:8580) and http: //www.ikir.ru:8280/lsaserver/MagneticPage.jsp. The development of the program system was supported by the grant of the RNF No. 14-1100194.

\section{Geomagnetic data analysis methods implemented in the software system}

\subsection{Estimate of geomagnetic disturbances intensity is based on the following operations:}

1. Wavelet transform of minute data is performed:

$$
W_{\Psi} f_{b, a}:=|a|^{-1 / 2} \int_{-\infty}^{\infty} f(t) \Psi\left(\frac{t-b}{a}\right) d t, f \in L^{2}(R), a, b \in R, a \neq 0,
$$


where $a$ is the scale, $\Psi$ is the basic wavelet.

In the decomposition (1.1) we use an orthonormal third-order Daubechies wavelet basis, which was determined by minimizing the error in data approximation [23]. Wavelet $\Psi$ is a window function and has a zero mean and, when the scale $a$ tends to be zero, coefficients $W_{\Psi} f_{b, a}$ describe the properties of the function $f$ in the neighborhood of $t=b$.

2. Then the intensity of field perturbations at time $t=b$ is estimated by the formula [24]:

$$
E_{b}=\sum_{a} e_{b, a}
$$

where $e_{b, a}=\left|W_{\Psi} f_{b, a}\right|$.

Fig. 1. illustrates the result of calculating the intensity of geomagnetic disturbances based on operation (1.2) for the period December 19-21, 2015.

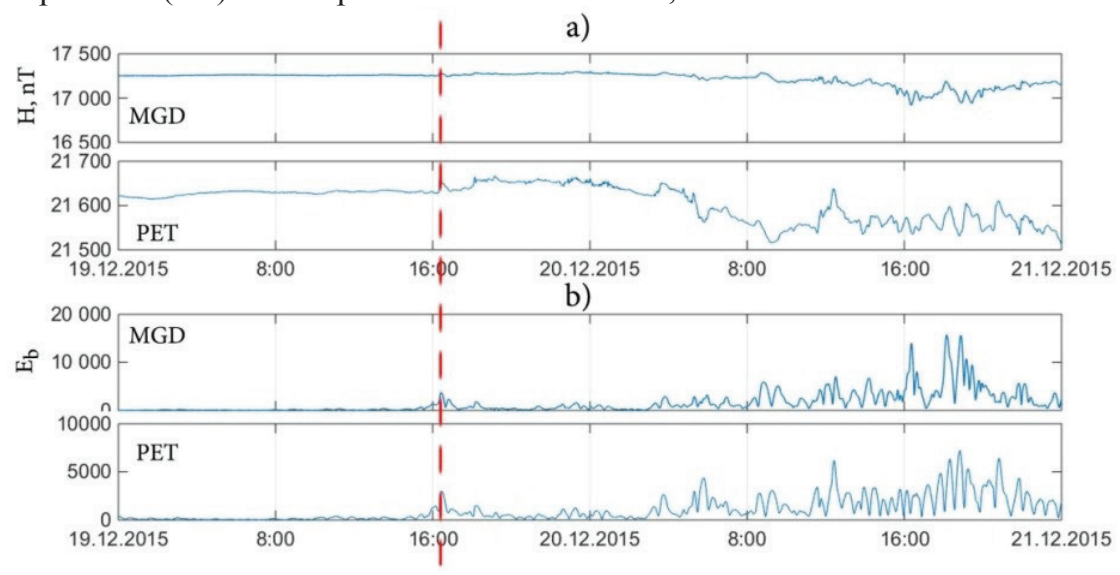

Fig. 1. The result of the operations (1.1), (1.2) for data stations "Magadan" and "Paratunka" during the period December 19-21, 2015; a) H-components of the Earth's magnetic field; b) calculation of the geomagnetic disturbances intensity, based on operation (1.2). The dashed line indicates the beginning of the magnetic storm

\subsection{Isolation of the periods of weak and strong geomagnetic activity based on the application of threshold functions:}

$$
K_{T_{a, 1}}\left(e_{b, a}\right)=\left\{\begin{array}{l}
0, \text { если } e_{b, a}<T_{a, 1} \\
e_{b, a}, \text { ссли } e_{b, a} \geq T_{a, 1}
\end{array}, K_{T_{a, 2}}\left(e_{b, a}\right)=\left\{\begin{array}{l}
0, \text { если } e_{b, a}<T_{a, 2} \\
e_{b, a}, \text { если } e_{b, a} \geq T_{a, 2}
\end{array},\right.\right.
$$

The threshold values of $T_{a, 1}$ allow us to identify weak and strong perturbations, and the threshold values of $T_{a, 2}$ allows us to identify strong perturbations. Identification of the thresholds $T_{a, 1}$ and $T_{a, 2}$ was performed by assessing a posteriori risk [25], the estimates used the data of the observatory "Paratunka" (Kamchatka region) for the period 2002-2010. The results of calculating the values of $T_{a, 1}$ and $T_{a, 2}$ for different scale levels $a$ are shown in Fig. 2. 


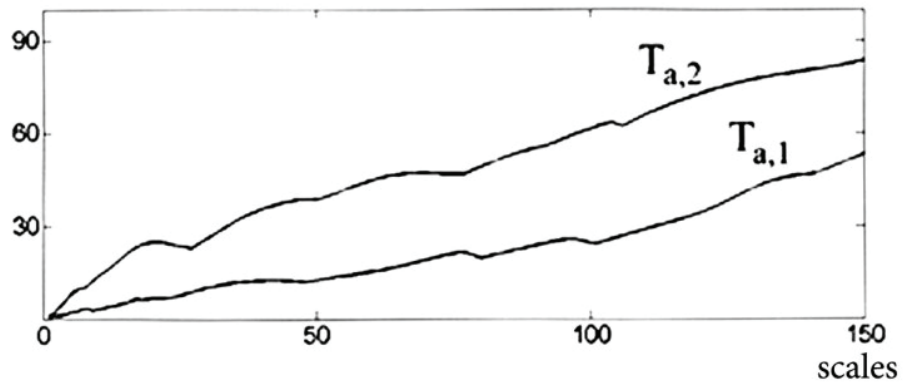

Fig. 2. The results of calculating the values of $T_{a, 1}$ and $T_{a, 2}$. On the axis oy, the values of $T_{a, 1}$ and $T_{a, 2}$ are shown

Fig. 3 shows the result of the application of threshold functions (1.3) to detect the periods of weak and strong geomagnetic activity for the period December 19-21, 2015.

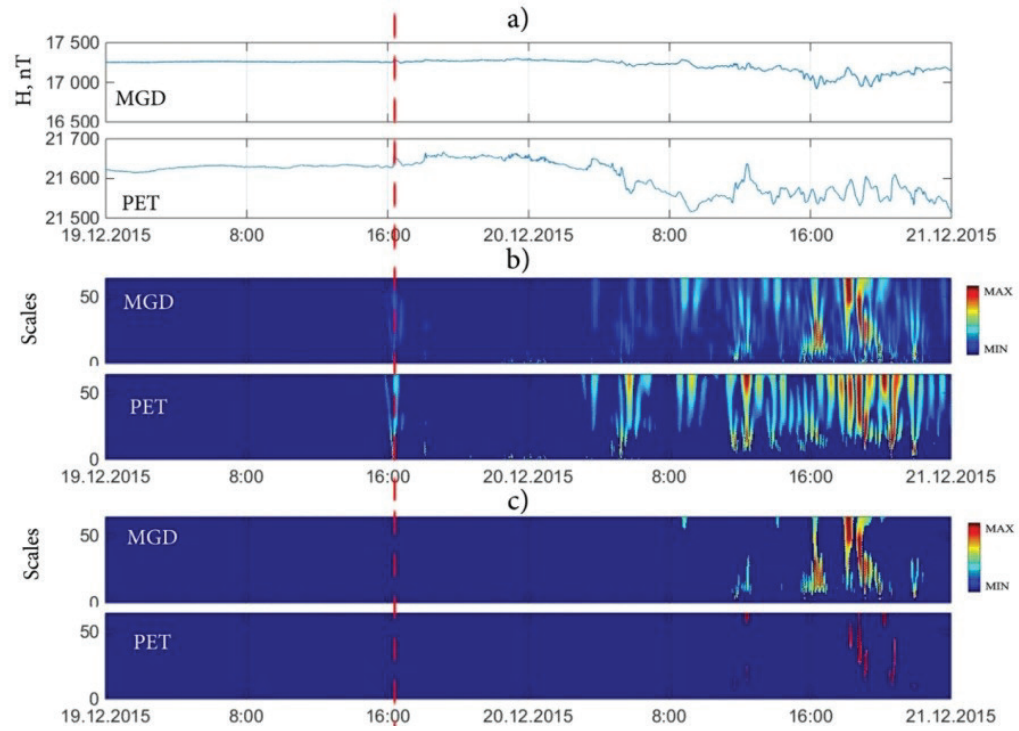

Fig. 3 Result of detection of periods of weak and strong geomagnetic activity according to the data of Magadan and Paratunka stations for the period December 19-21, 2015. a) H-components of the Earth's magnetic field; b) detected periods of weak and strong geomagnetic activity (the threshold $T_{a, 1}$ was applied); c) detected periods of strong geomagnetic activity (the threshold $T_{a, 2}$ was applied). The dashed line indicates the beginning of the magnetic storm

The software system supports the generally accepted IAGA-2002 data format and uses minute data for the processing. Fig. 4 presents the results of the minute data analysis of Paratunka and Magadan stations during the magnetic storm on August 15, 2015. According to space weather data (http://ipg.geospace.ru/) on August 15, 2015, a sharp increase in the solar wind speed was registered, caused by the coronal ejection of solar matter (CME from August 12) and high-speed solar wind from the coronal hole (reached the central meridian on August 11). At "Paratunka" and "Khabarovsk" stations there were sharp increases in the horizontal component of the field at 08:30 UT (Fig. 4a). Analysis of the geomagnetic disturbances intensity (Fig. 4b) shows that, approximately 12 hours before the beginning of the magnetic storm, short-term increases were observed. During the main phase of the 
storm (the period of a significant decrease in the Dst-index), the maximum values of the geomagnetic disturbances intensity were recorded (Fig. 4b). This example shows the effectiveness of the proposed software for geomagnetic data analysis tasks, which, in contrast to the analogues, allows obtaining accurate quantitative estimates of the degree of geomagnetic field disturbance.

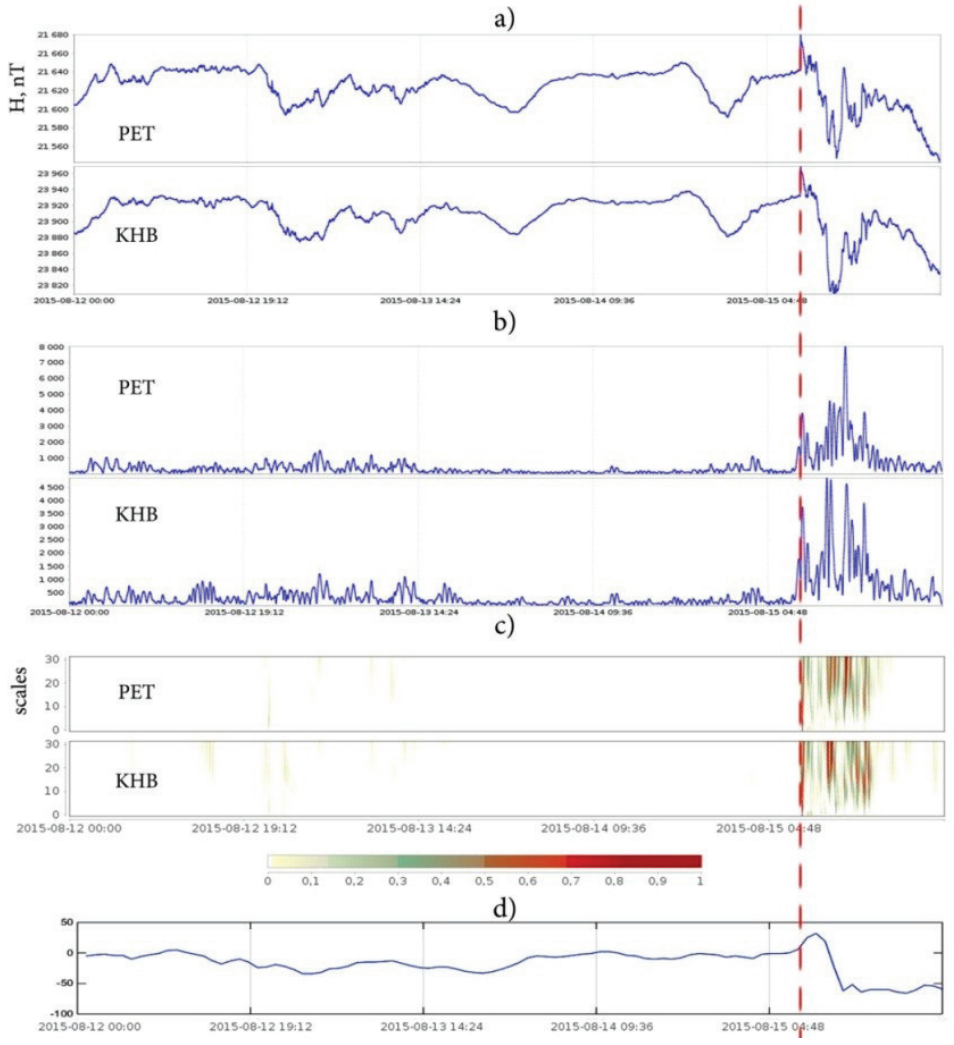

Fig. 4 Software processing of minute geomagnetic data during the period August 12-15, 2015 a) Hcomponents of the Earth's magnetic field; b) calculation of the geomagnetic disturbances intensity, based on the operation $(1.2)$; c) detected periods of weak and strong geomagnetic activity (the threshold $T_{a, 1}$ was applied); d) Dst- index. The dashed line indicates the beginning of the magnetic storm

\subsection{Estimation of the geomagnetic disturbances intensity in the range of geomagnetic pulsations}

According to (1.2), the intensity of positive (increase in field variation relative to the characteristic level) and negative (decrease in the variation of the geomagnetic field with respect to the characteristic level) geomagnetic disturbances on the scale at a time point $t=b$

$$
e_{b, a}^{ \pm}=\left(W_{\Psi} f\right)(b, a)
$$

Then the intensity of the field disturbance at a time point $t=b$ can be estimated: 


$$
E_{b}^{ \pm}=\sum_{a=a_{1}}^{a_{2}} e_{b, a}^{ \pm}
$$

Fig. 5 shows the result of an estimate of the geomagnetic disturbances intensity in the range of $\mathrm{Pi} 2$ pulsations (periods from 45 to 150 seconds) based on operation (1.5) within the period from 7:00 UT to 10:00 UT on August 15, 2015.
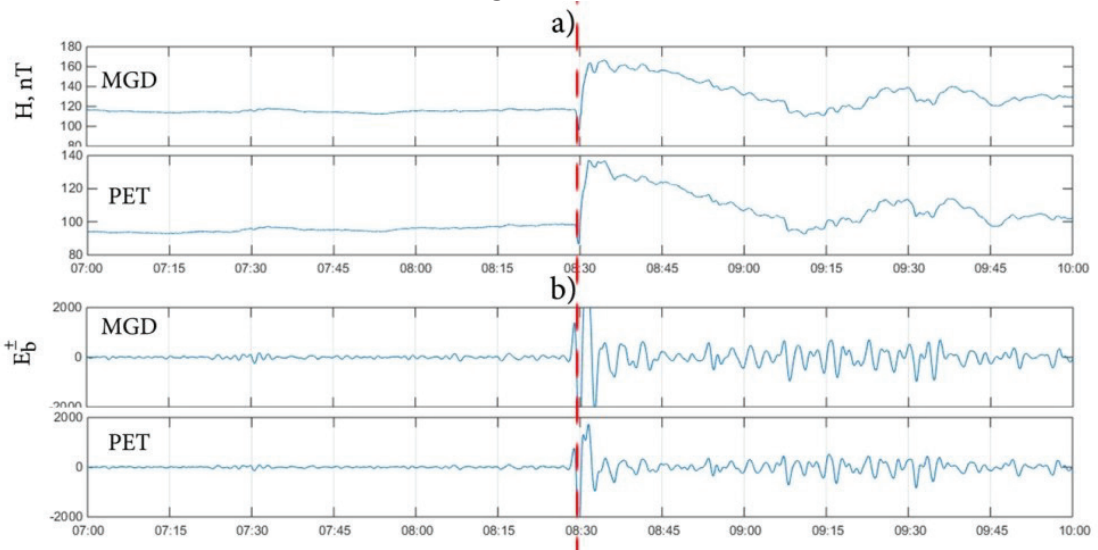

Fig. 5 The result of estimation of geomagnetic disturbances intensity in the range of Pi2 pulsations at Magadan and Paratunka stations on August 15, 2015 from 7:00 UT to 10: 00 UT. a) H-components of the Earth's magnetic field; b) calculation of geomagnetic disturbances intensity, based on the operation (1.5). The dashed line indicates the beginning of the magnetic storm

Geomagnetic disturbances in the range of pulsations are extracted on the basis of decomposition of variations of the horizontal component of the geomagnetic field into wavelet packets:

$$
f_{(0,0)}(t)=\sum_{n} c_{j, k, n} \varphi_{j, k, n}(t)+\sum_{(j, k) \in P_{i}} \sum_{n} d_{j, k, n} \Psi_{j, k, n}(t)+\sum_{(j, k) \notin P_{i}} \sum_{n} d_{j, k, n} \Psi_{j, k, n}(t),
$$

where $\varphi_{j, k}=\left\{\varphi_{j, k, n}\right\}_{n \in Z}$ is basis in the node $(j, k)$ of a wavelet packet tree obtained by a scaling function; $\Psi_{j, k}=\left\{\Psi_{j, k, n}\right\}_{n \in Z}$ is the wavelet-basis in the node $(j, k)$ of the wavelet packets tree; coefficients $c_{j, k, n}=\left\langle f, \varphi_{j, k, n}\right\rangle ; d_{j, k, n}=\left\langle f, \Psi_{j, k, n}\right\rangle ; P_{i}$ is a set of index pairs, $(j, k)$ is a node of the wavelet packet tree, where $j$ is the scale, $\mathrm{k}$ is the component number, the subscript $(0,0)$ indicates the origin of the discrete data.

Approximating component $\sum_{n} c_{j, k, n} \varphi_{j, k, n}(t)$ describes the trend of the geomagnetic field variation, detailing components $\sum_{(j, k) \in P_{i}} \sum_{n} d_{j, k, n} \Psi_{j, k, n}(t)$ describe pulsations from a range determined by a set of indexes $P_{i}$. Component $\sum_{j \notin D_{i}} \sum_{n} d_{j, k, n} \Psi_{j, k, n}(t)$ is taken as noise. Fig. 6 shows the scheme of decomposition into wavelet packets up to the 2nd level. 


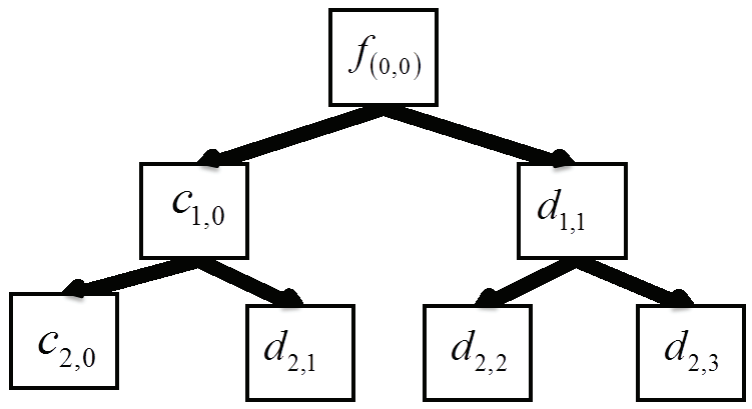

Fig. 6. Scheme of decomposition $f_{(0,0)}(t)$ into wavelet packets

In order to extract the pulsations in the ranges Pc1, Pc2, Pc3, Pc4, Pc5 we use the following sets of index pairs: $P_{1}^{P c 1}=\{(1,1) ;(3,3)\}, P_{2}^{P c 2}=\{(3,2)\}, P_{3}^{P c 3}=\{(3,1) ;(4,1),(6,3)\}$, $P_{4}^{P_{c 4}}=\{(6,2) ;\}, P_{5}^{P c 5}=\{(7,1) ;(8,1),(10,3)\}$ and to extract the pulsations Pi1, Pi2, Pi3 we use $P_{6}^{P i 1}=\{(1,1) ;(2,1) ;(2,2) ;(3,1) ;(4,1) ;(5,1)\}, P_{7}^{P i 2}=\{(6,1) ;(7,1)\}, P_{3}^{P i 3}=\{(8,1) ;(9,1)\}$, respectively.

\subsection{Construction of a wavelet-portrait of a magnetic storm:}

1. According to station network data on the basis of operation (1.2), geomagnetic disturbances intensity is calculated;

2. On the basis of operation (1.1) a wavelet spectrum of geomagnetic disturbance intensity values is constructed.

The second geomagnetic data of the ground station network are used to construct the wavelet-portrait. The obtained geomagnetic disturbances intensity wavelet-spectra show the disturbance distribution dynamics in the analyzed regions.

Fig. 7 shows the result of constructing a wavelet-portrait during the magnetic storm on August 15, 2015. The constructed wavelet-portrait shows a spatial-temporal pattern of the disturbance dynamics before and during the magnetic storm along the meridian of the observations. In the initial phase of the storm from 8:00 UT to 9:30 UT, disturbances were observed in a wide range of frequencies at all stations. During the main phase of the storm from 10:00 UT, the disturbances at MGD, PET, MMB stations were of a general nature, which characterizes large-scale processes in the magnetosphere. At the high-latitudinal KTN station, the maximum disturbances were observed within the period from 11:20 UT to 15:00 UT and coincided with the maximum values of the indices AE, AU, AL. Moreover, within the period from 12:30 UT to 13:10 UT, active regions were detected at the northern stations of KTN and MGD (marked by the oval in Fig. 7). They are likely to be associated with auroral processes. 


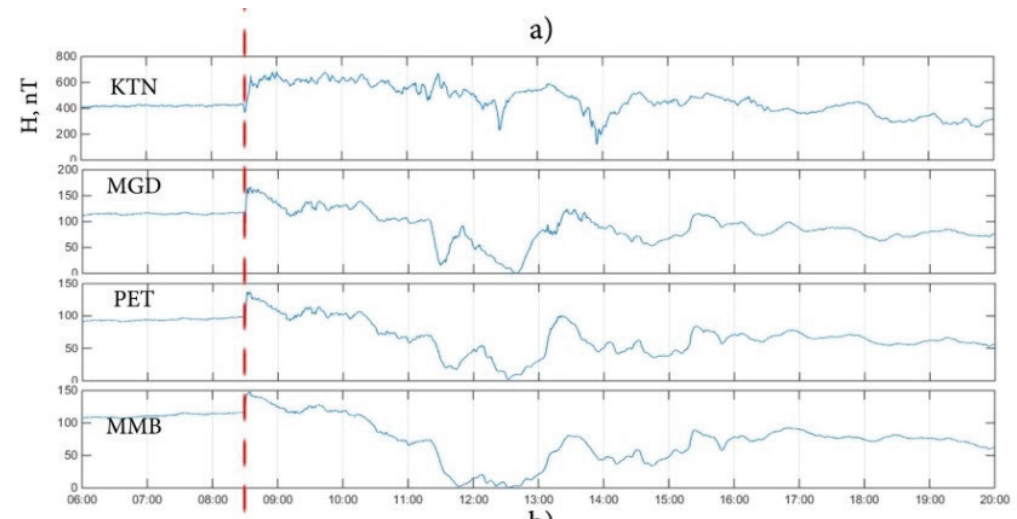

b)
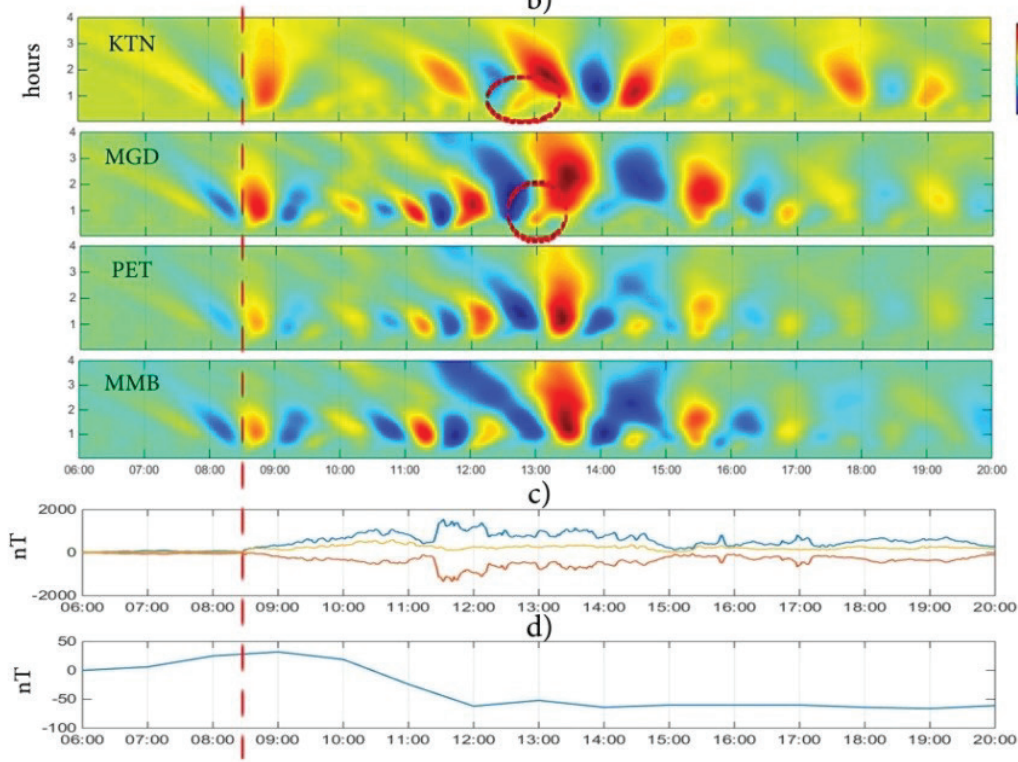

Fig. 7. Second Earth magnetic field data processing by the «Aurora» system during the magnetic storm on August 15, 2015 a) H-components of the Earth's magnetic field; b) wavelet-spectrum of the geomagnetic disturbances intensity; c) indices AE (blue line), AU (yellow line) and AL (red line); d) Dst- index. The dashed line indicates the beginning of the magnetic storm

\section{Conclusion}

The paper presents a software system for geomagnetic data processing and analysis that allows us to perform the detailed analysis of the occurrence and dynamics of geomagnetic field perturbations in the areas under consideration during the periods of increased solar activity. The implemented software modules are available in the Internet (http://aurorasa.ikir.ru:8580, http://www.ikir.ru:8280/lsaserver/MagneticPage.jsp). Comments and suggestions on the application of the software can be sent to aurora@ikir.ru.

The work was supported by the RNF Grant No. 14-11-00194. The author expresses gratitude to the organizations that are registering the geomagnetic data that were used in the work and to the Institute of Applied Physics for the continuous implementation and publication of a space weather survey in the Internet. 


\section{References}

1. Yu.I. Yermolaev, M.Yu. Yermolaev, IZV ATMOS OCEAN PHY+, 46(7), 799-819 (2010)

2. R.I. Krasnoperov, A.A. Soloviev, Gornyi Zhurnal 10, 89-93, doi: 10.17580/gzh.2015.10.16 (2015)

3. O.V. Mandrikova, E.A. Zhizhikina, Computer Optics, 39(3), 420-428 (2015, in russian)

4. O.V. Mandrikova, N.V. Fetisova (Glushkova), Riad Taha Al-Kasasbeh, D.M. Klionskiy, V.V. Geppener, M.Y. Ilyash, Annals of geophysics 58(5), (2015)

5. O.V. Mandrikova, N.V. Glushkova, Yu.A. Polozov, Pattern Recognition and Image Analysis, 25(3), 470-480, 2015.

6. O.V. Mandrikova, T.L. Zalyaev,11th International Conference "Problems Of Geocosmos", 77-78 (2016)

7. S.Yu. Khomutov, VarSITI Newletter (2014)

8. S.Yu. Khomutov, I.N. Khomutova, Vestnik KRAUNC. Fiz.-mat. nauki. 15(4), 112117. (2016) (In Russian)

9. R.V. Sidorov, A.A Soloviev, Sh.R. Bogoutdinov, Izvestiya, Physics of the Solid Earth, 48(5), 410-414, (2016)

10. A.A Soloviev et al. Data Science Journal, 12 doi:10.2481/dsj.WDS-019 (2013)

11. Soloviev, A. Chulliat, S. Bogoutdinov, A. Gvishiani, S. Agayan, A. Peltier, B. Heumez, EPS, 64(9), 743-752, (2012)

12. Daubechies Ten Lectures on Wavelets (CBMS-NSF Lecture Notes nr. 61, 1992)

13. A.G. Hafez, E. Ghamry, H. Yayama, K. Yumoto, ASR, 51, 39-49 (2013)

14. Z. Xu, L. Zhu, J. Sojka, P. Kokoszka, A. Jach, J. Atmos. Solar-Terr. Phys., 70, 15791588 (2008)

15. Jach, P. Kokoszka, J. Sojka, L. Zhu, J. Geophys. Res., 111(A9) doi:10.1029/2006JA011635 (2006)

16. O.V. Mandrikova, S.E. Smirnov, I.S. Solov'ev, Geomagn. Aeron., 52(1), 111-120 (2012)

17. V.P. Golovkov, V.O. Papitashvili, N.E. Papitashvili, Geomagn. Aeron., 29, 667-670 (1989)

18. K. Nowożyński, T. Ernst, J. Jankowski, Geophys. J. Int., 104, 85-93 (1991)

19. M. Menvielle, N. Papitashvili, L. Hakkinen, C. Sucksdorff, Geophys. J. Int., 123, 866886. (1995)

20. K. Unnikrishnan, Journal of Atmospheric and Solar-Terrestrial Physics, 121, 206-220 (2014)

21. K. Alielden, A. Mahrous, Solar Radio (CESRA 2016), 89 (2016)

22. O.V. Mandrikova, E.A. Zhizhikina, V.V. Geppener, XVIII International Conference On Soft Computing and Measurements,(2015)

23. O.V. Mandrikova, I. Solovjev, V.V. Geppenerc, A-KR. Taha, D. Klionskiy, DIGIT SIGNAL PROCESS, 23, 329-339 (2013)

24. O.V. Mandrikova, I.S. Solovev, T.L. Zalyaev, EPS, 66. doi:10.1186/s40623-014-01480 (2014)

25. B. R. Levin Theoretical Basis of Statistical Radio Techniques (Fizmatgiz Moscow, 1963). 